\title{
Editorial
}

Frédéric Lagarce*

\section{After Ten Issues Our Journal Has Found Its Audience and Main Topics}

https://doi.org/10.1515/pthp-2018-0024

Since "Pharmaceutical Technology in Hospital Pharmacy" was launched in March 2016, we have published ten issues divided into three volumes. This corresponds to more than 50 research, opinion and review papers, plus conference reports, editorials and so on. You are now holding the 11th issue in your hands, which will also be distributed during the 21st GERPAC European meeting in October 2018. It highlights the main topics of our journal and obviously those of the GERPAC for more than 20 years: automation, chemical contamination, stability studies, feedback on professional practice and vision for the future of pharmaceutical technology in hospitals.

The first three papers are from the field of analytical chemistry. U. Bihola et al. from Gujarat in India describe the Quality by Design ( $\mathrm{QbD})$ approach for the simultaneous detection of two drugs (torsemide and spironolactone) and their impurities. This paper is a good example for how to set-up and validate an analytical method using $\mathrm{QbD}$, which is not found very often. This method also highlights the use of LC/MS for stability studies. It determines the performance of the technique to detect and quantify the degradation products after stress conditions. Two further original stability studies are presented in this issue. The study presented by the team of Jean Vigneron in Nancy, France (first author Elise D'Huart) is interesting because it is performed on a very small molecule, i.e. sodium thiosulfate. This is not very common and certain particularities are to be taken into account: the degradation pathways or the detection of the molecule and its degradation products. The first study on sodium thiosulfate dates back to 1923 and there is still some new data to accumulate - refreshing! The last paper on this subject is from the team of J.D. Hecq from CHU Ste Godine in Belgium. This paper describes a long term comparison of the stability of an original antibiotic infusion in polyolefin bags and its generic. This study is important as

*Corresponding author: Frédéric Lagarce, Department of Pharmacy, University of Angers, CHU Angers, 4 rue Larrey, 49033 Angers, France, E-mail: frederic.lagarce@univ-angers.fr generics should only demonstrate bioequivalence i.e. a non-significant difference between the bioavailability of the princeps and the generic formulation. It is thus possible that the stability varies between the two presentations of the drug after reconstitution in the pharmaceutical environment. In the choices of generic, stability equivalence is then worth studying. This was done by the team of Prof. J.D. Hecq after microwave freeze thaw treatment, which was one of the innovations proposed by the team a few years ago in order to allow a longer stability of reconstituted drug products. The paper concludes that no differences between the princeps and the generic have been found if stability after reconstitution is concerned.

The second part of this issue is dedicated to automation. In a first study, M. Frederici, I. Krämer and R. Schierl from Mainz and Munich in Germany, provide some very interesting data on surface contamination (monitoring Platinum and 5-FU traces) during the use of two robotic systems, namely APOTECAchemo from Loccioni Group in Italy and Cytocare from Health Robotics, Italy. This was compared to manual preparation in a regular biological safety cabinet in Mainz hospital facilities. Syringes and infusion bags were also sampled. The chemical contamination was found to remain in the robot area. Moreover the automatically prepare infusion bags were less contaminated than those that were manually prepared. The study emphasizes the importance of implementing optimised cleaning procedures in order to minimise contamination. We believe that this study will be a first reference in the field of chemical contamination in automated compounding areas. The second paper is from the team of P. Bonnabry in Geneva, Switzerland (first author L. Carrez). It describes the performance qualification of another automated system (Pharmahelp, Fresenius Kabi, Germany). The main interest of this paper is to provide a standard plan to perform qualification of any automated compounder. This is thus very useful for the readers of our journal.

In the last section of this issue you will find two opinion papers. The first one from J. Sorrieul et al (Angers, France) is a feedback on professional practice and circuit organisation of the preparation and delivery of intrathecal analgesics in an oncology clinic. Pain 
control is an important part of cancer treatment. This is why it is of high value for the patient to benefit from such an organisation, which will provide the compounded intrathecal preparation of morphine, ziconotide and other analgesics in good delay with a controlled quality.

The last paper of this issue is very special. It is the personal view of a very experienced pharmacist, Prof. A.T. Florence, on the importance of pharmaceutical technology nowadays in hospitals. During his long career Prof. Florence was involved in research at the highest level. He worked in the fields of pharmaceutical technology, nanomedicine, biopharmaceutics and cancer therapy, just to name a few. His view on the role of technology and its relation to the clinical field is worth reading and we really hope that it will inspire the next generation of hospital pharmacists, as it was the case for us during the 90 s and the beginning of this century. In the name of the whole editorial board we really want to thank Prof. Florence for sharing his views on our field.

As you can see this 11th issue of our journal is of high quality, with original research, novel data and opinions. We really hope that you will like it as much as we did. We also announce here that the next special issue (to be published in 2019) will be dedicated to automation in hospital pharmacies. Submissions are possible until December 30th. Finally, as a recognition of the growing quality of the journal, we would like to let you know that PTHP is now indexed in Elsevier's Embase $^{\circledR}$ database. 